\title{
Factors Associated With Neurogenic Bowel Dysfunction Severity in Spinal Cord Injury: A Cross-sectional Study
}

\author{
Ida Mohammadi ${ }^{\circledR}$, Mohammadhosein Akhlaghpasand ${ }^{\# *}{ }^{(0)}$, Roozbeh Tavanaei ${ }^{\circledR}$, Maryam Golmohammadi, \\ Alireza Zali ${ }^{\circledR}$, Saeed Oraee-Yazdani ${ }^{(\mathbb{}}$
}

Shohada Tajrish Neurosurgical Center of Excellence, Functional Neurosurgery Research Center, Shohada Tajrish Hospital, Shahid Beheshti University of Medical Sciences, Tehran, Iran

\begin{abstract}
Background: Neurogenic bowel dysfunction (NBD) caused by spinal cord injury (SCl) is a major life limiting issue for many patients. However, studies detailing the factors contributing to bowel problems are scarce. The aim of this cross-sectional questionnaire-based study was to evaluate the associations between different aspects of bowel problems and characteristics of $\mathrm{SCl}$, such as time since injury (TSI), level of injury (LOI), and the cause of injury.

Methods: Two questionnaires (the NBD score questionnaire and the Cleveland clinic constipation system score questionnaire) were administered to complete, traumatic patients with $\mathrm{SCl}$ that matched the designated criteria. The total scores and the subscales were then regressed to TSI and LOI, and the distribution patterns of NBD score severity according to LOI and cause of injury were shown in a diagram.

Results: Within the subscales of the NBD score, frequency of defecation, digital stimulation or evacuation of the anorectum, and perianal skin problems were all significantly and positively correlated with the TSI, yet the frequency of fecal incontinence was negatively associated with this variable. Moreover, uneasiness, headache, or perspiration during defecation and regular use of drops against constipation were negatively associated with the LOI. In the diagrams, patterns of NBD score severity were similar according to LOI, but visibly differed according to cause. With respect to the CCCS score (Cleveland Clinic Constipation Scoring System), the score itself was shown to be positively associated with TSI. Within the subscales, difficulty, time spent in lavatory, and duration of constipation were positively correlated with TSI. Furthermore, type of assistance was negatively associated with the LOI.

Conclusion: Different characteristics of $\mathrm{SCI}, \mathrm{TSI}, \mathrm{LOI}$, and cause, each are significantly and distinctly associated with different aspects of the bowel problems that patients with $\mathrm{SCl}$ face.

Keywords: Spinal cord injury; Neurogenic bowel dysfunction; Cleveland Clinic Constipation Scoring System.
\end{abstract}

\author{
*Correspondence to \\ Mohammadhosein \\ Akhlaghpasand, Shohada \\ Tajrish Neurosurgical Center \\ of Excellence, Functional \\ Neurosurgery Research Center, \\ Shohada Tajrish Hospital, \\ Shahid Beheshti University \\ of Medical Sciences, Tehran, \\ 1988873554, Iran. Tel: \\ +9821-22438023, Fax: +9821 \\ 22438023; \\ Email: Akhlaghpasandm@ \\ yahoo.com
}

\#These authors contributed equally to this work.

Published online 30 October 2021

Citation: Mohammadi I, Akhlaghpasand M, Tavanaei R, Golmohammadi M, Zali A, Oraee-Yazdani S. Factors associated with neurogenic bowel dysfunction severity in spinal cord injury: a cross-sectional study. Clin Neurosci J. 2021;8(4):168-174. doi:10.34172/ icnj.2021.34

\section{Introduction}

Spinal cord injury (SCI) is a devastating neurological condition that produces a wave of unfortunate ramifications, affecting most, if not all organs. It is estimated that each year approximately 12000 new cases of SCI occur in the United States, with about 300000 patients living with neurological consequences. ${ }^{1}$ When the spinal cord incurs direct mechanical trauma, local ischemia and hemorrhage occur, leading to edema and neural cell death in the immediate vicinity. ${ }^{2}$ Afterwards, changes in the injury zone result in the breakdown of cellular membranes, and, consequently, the release of inflammatory cytokines, all leading to an even greater extent of neural cell loss. ${ }^{3}$ When SCI reaches the chronic stage, a cystic cavity forms that is surrounded by an astroglial scar, and axons distal to the site of injury undergo progressive Wallerian degeneration. ${ }^{4}$ The astroglial scar and cystic cavities compounded with less than sufficient endogenous remyelination and axonal regrowth severely limit the potential of the spinal cord for intrinsic repair. ${ }^{5}$ Hence, the end result of the pathophysiological changes in the spinal cord includes permanent deficits such as loss of sensory perception and motor paralysis at the distal level of the injury. ${ }^{6}$ SCI affects nearly every aspect of the lives of the patients and their families, since loss of independence and elevated lifelong mortality rates follow SCI. ${ }^{7}$

(C) 2021 The Author(s). This is an open access article distributed under the terms of the Creative Commons Attribution License (http:// creativecommons.org/licenses/by/4.0/), which permits unrestricted use, distribution, and reproduction in any medium, provided the original work is properly cited. 
When a patient is affected by SCI, the damages to the sensory and motor systems are conspicuous, yet the impact of the injury on the willful elimination of stool and urine usually goes unnoticed by the general population. ${ }^{8}$ In the aftermath of the injury, the patients are faced with neurogenic bowel dysfunction (NBD), since SCI affects colorectal motility, ${ }^{9}$ transit times,${ }^{10}$ and bowel emptying, ${ }^{11}$ often causing constipation, fecal incontinence, or a combination of both. ${ }^{12}$ However, it should be noted that the effects of neurogenic bowel on the quality of life after SCI extend far well beyond the mere inconvenience or the pain. In the Stockholm SCI study, bowel dysfunction was rated as a moderate to severe life-limiting problem by $41 \%$ of the patients. ${ }^{13}$ In a study that evaluated the physical and psychological effects of SCI, on a scale of 0 (for no perceived problem) to 10 (maximum perceived problem), patients rated their loss of mobility as a mean of 6.8 and their bowel management as 5.1. ${ }^{14}$ Patients with SCI commonly declare incontinence as the reason for not engaging in outside activities. ${ }^{8}$ Additionally, an immense amount of time, expense, and attendant support are needed to cope with the secondary outcomes of SCI, including bowel problems. ${ }^{15}$ Yet, despite all the disastrous impacts of NBD on the patient's life, a limited number of studies have been dedicated to identifying the influence that each of the characteristics of the injury exerts on different aspects of bowel problems in patients with SCI.

To better evaluate the factors contributing to the extent of the problems associated with the neurogenic bowel, we aimed to study the correlations between the severity of the problems and variables such as the cause of injury, the level of injury (LOI), and the time since injury (TSI).

\section{Materials and Methods}

This study was cross-sectional and questionnaire-based. A hundred and ten patients were enrolled in the study according to the selection criteria. All patients were in the 18-65 age range, had complete traumatic SCI (ASIA classification A), with either a cervical or thoracic LOI. Exclusion criteria were as follows: 1) pre-existing comorbidities such as diabetes mellitus and cardiovascular diseases, 2) disorders and conditions involving the limbs such as deformities and amputations, and 3) neurological and neurodegenerative conditions such as multiple sclerosis, cerebral palsy, spinal muscular atrophy, etc.

Two questionnaires were administrated to the patients: the NBD score questionnaire, and the Cleveland Clinic constipation system (CCCS) score questionnaire. The NBD score (range, 0- 47, with 47 representing severe symptoms) is a validated symptom score where each symptom of NBD is weighted concerning its impact on quality of life. In this scale, four categories have been defined based on the severity of dysfunction: very minor
NBD (0-7), minor NBD (7-9), moderate NBD (9-13), and severe NBD (14 or more). ${ }^{12}$ The CCCS score (range, $0-30$, with 30 representing severe symptoms) is mainly aimed at the evaluation of constipation. It correlates well with objective physiological findings in constipated patients and allows uniformity in the assessment of the severity of constipation. ${ }^{16}$

At inclusion, demographic data were obtained and the questionnaires were administered. Univariate linear regression was then used to assess both the presence and the extent of correlation of each of the subscales of the two scoring systems with the LOI and the interval passed since SCI. $P<0.05$ were considered statistically significant. Different severities of the NBD score were also presented based on the cause of SCI and also the LOI so that the possible correlations could be better observed. Data were analyzed using SPSS software, version 16.0 (IBM Crop., Armonk, USA).

\section{Results}

\section{Demographics}

We included 110 patients were included in the present study. The mean age of the patients was $29.49 \pm 8.45$ and a mean interval of $21.25 \pm 17.46$ years had passed since the injury. Most of the enrolled patients were male (71.8\%) and also most of them suffered from thoracic SCI (73.6\%). Other demographic characteristics can be seen in Table 1.

\section{NBD Score}

Descriptive Results

The items in the questionnaire, plus the mean score $(\mu)$ and the standard deviation $(\sigma)$ of each were as follows: Frequency of defecation $(\mu=2.38, \sigma=2.42)$; Time used for each defecation $(\mu=1.71, \sigma=2.66)$; Uneasiness, headache or perspiration during defecation $(\mu=0.90$, $\sigma$ $=1.00)$; Regular use of tablets against constipation $(\mu=$ $0.87, \sigma=1.00$ ); Regular use of drops against constipation $(\mu=0.22, \sigma=0.63)$; Digital stimulation or evacuation of

Table 1. Demographic Results

\begin{tabular}{lccccc}
\hline \multirow{2}{*}{ Age } & & Mean & $\begin{array}{c}\text { Standard } \\
\text { Deviation }\end{array}$ & No. & $\%$ \\
\hline \multirow{2}{*}{ Gender } & Male & 29.49 & 8.45 & - & - \\
& Female & - & - & 79 & $71.8 \%$ \\
\multirow{2}{*}{ Cause } & Accident & - & - & 31 & $28.1 \%$ \\
& Falling & - & - & 76 & $69.1 \%$ \\
\multirow{2}{*}{ Interval from SCl } & Others & - & - & 30 & $27.3 \%$ \\
\multirow{2}{*}{ LOI } & Cervical & - & - & 4 & $3.6 \%$ \\
& Thoracic & - & - & 81 & $73.6 \%$ \\
\hline
\end{tabular}

Abbreviations: $\mathrm{SCl}$, spinal cord injury; LOI, level of injury. 
the anorectum $(\mu=3.93, \sigma=2.87)$; Frequency of fecal incontinence $(\mu=5.91, \sigma=4.49)$; Medication against fecal incontinence $(\mu=0.36, \sigma=1.16)$; Flatus incontinence ( $\mu$ $=1.51, \sigma=0.86)$; Perianal skin problems $(\mu=0.55, \sigma=$ 1.16). The mean \pm SD total NBD score was $18.09 \pm 7.59$ (Table 2).

\section{Regression to Time Since Injury}

Based on the regression analysis, the total NBD score per se was not significantly associated with the TSI, yet associations were observed among the subscales. Frequency of defecation ( $\mathrm{B}=0.265, P=0.006$ ), digital stimulation or evacuation of the anorectum $(\mathrm{B}=0.185, P=$ $0.049)$, and perianal skin problems $(B=-0.209, P=0.029)$ were all positively correlated with TSI. On the other hand, the frequency of fecal incontinence $(\mathrm{B}=0.258, P=0.006)$ was negatively associated with this variable (Table 2 ).

\section{Regression to the Level of Injury}

We found no significant associations between the total NBD score and the LOI. Within the subscales, uneasiness, headache, or perspiration during defecation $(B=-0.288$, $P=0.003)$ and regular use of drops against constipation $(\mathrm{B}=-0.292, P=0.002)$ were negatively associated with LOI (Table 2).

\section{Severity According to the Level of Injury}

When patients were grouped according to the severity of SCI, we observed that among the cervical patients, the severity of $\mathrm{NBD}$ was very minor in $16.7 \%$, minor in $50.0 \%$, and moderate in $33.3 \%$ of the cases. Among the thoracic patients, the severity was very minor in $15.0 \%$, minor in $45.0 \%$, moderate in $30.0 \%$, and major in $10.0 \%$ of the cases (Figure 1A).

\section{Severity According to the Cause}

Among the patients that fall victim to SCI as a result of accidents, the severity was very minor in $17.6 \%$, minor in $41.2 \%$, moderate in $29.4 \%$, and major in $11.8 \%$ of the cases. When falling is the cause of the SCI, the NBD severity is very minor, minor, and moderate in $11.1 \%$, $77.8 \%$, and $11.1 \%$ of the patients, respectively. $100 \%$ of the SCI patients injured by other causes have moderate SCI (Figure 1B).

\section{CCCS Score}

Descriptive Results

The items in the questionnaire, plus the mean score $(\mu)$ and the standard deviation $(\sigma)$ of each were as follows: Frequency of bowel movements $(\mu=1.06, \sigma=0.78)$; Difficulty: painful evacuation effort $(\mu=1.90, \sigma=1.50)$; Completeness: feeling incomplete $(\mu=1.92, \sigma=1.31)$; Pain: abdominal pain $(\mu=1.46, \sigma=1.43)$; Time: minutes in lavatory per attempt $(\mu=1.90, \sigma=1.54)$; Assistance: type of assistance $(\mu=1.08, \sigma=0.93)$; Failure: unsuccessful attempts for evacuation per 24 hours $(\mu=0.83, \sigma=1.35)$; History: duration of constipation $(\mu=0.38, \sigma=0.63)$. The mean \pm SD total CCCS score was $10.46 \pm 5.36$ (Table 3 ).

\section{Regression to the Time Since Injury}

When this score is regressed to TSI, a significant positive association can be observed $(\mathrm{B}=0.279, P=0.044)$. Within

Table 2. Neurogenic Bowel Dysfunction Results

\begin{tabular}{|c|c|c|c|c|c|c|}
\hline & \multicolumn{2}{|c|}{ Descriptive Results } & \multicolumn{2}{|c|}{$\begin{array}{c}\text { Univariate Linear Regression } \\
\text { to TSI }\end{array}$} & \multicolumn{2}{|c|}{$\begin{array}{c}\text { Univariate Linear Regression } \\
\text { to LOI } \\
\end{array}$} \\
\hline & Mean & SD & $\begin{array}{c}\text { Standardized } \\
\text { Coefficients (Beta) }\end{array}$ & $\boldsymbol{P}$ & $\begin{array}{c}\text { Standardized } \\
\text { Coefficients (Beta) }\end{array}$ & $P$ \\
\hline Frequency of defecation & 2.38 & 2.42 & 0.265 & $0.006^{* *}$ & -0.071 & 0.477 \\
\hline Time used for each defecation & 1.71 & 2.66 & -0.056 & 0.566 & 0.013 & 0.894 \\
\hline $\begin{array}{l}\text { Uneasiness, headache or perspiration during } \\
\text { defecation }\end{array}$ & 0.90 & 1.00 & 0.115 & 0.238 & -0.288 & $\mathbf{0 . 0 0 3}^{* *}$ \\
\hline Regular use of tablets against constipation & 0.87 & 1.00 & -0.001 & 0.989 & -0.040 & 0.676 \\
\hline Regular use of drops against constipation & 0.22 & 0.63 & -0.507 & 0.613 & -0.292 & $0.002^{* *}$ \\
\hline Digital stimulation or evacuation of the anorectum & 3.93 & 2.87 & 0.185 & $0.049^{*}$ & -0.153 & 0.115 \\
\hline Frequency of fecal incontinence & 5.91 & 4.49 & -0.209 & $0.029^{*}$ & 0.173 & 0.077 \\
\hline Medication against fecal incontinence & 0.36 & 1.16 & 0.108 & 0.260 & 0.027 & 0.781 \\
\hline Flatus incontinence & 1.51 & .86 & -0.150 & 0.115 & -0.120 & 0.217 \\
\hline Perianal skin problems & 0.55 & 1.16 & 0.258 & $0_{0.006}^{* *}$ & -0.072 & 0.457 \\
\hline NBD Total Score & 18.09 & 7.59 & 0.066 & 0.491 & -0.042 & 0.666 \\
\hline
\end{tabular}

Abbreviations: SCl, spinal cord injury; TSI, time since injury; LOI, level of injury; NBD, neurogenic bowel dysfunction.

$*: P$ value $<0.05 ; * *: P$ value $<0.01$ 


\section{Severity of NBD and LOI}

$100.00 \%$

$80.00 \%$

$60.00 \%$

$40.00 \%$

$20.00 \%$

$0.00 \%$

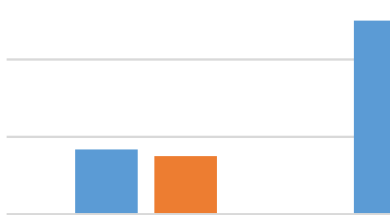

Very minor

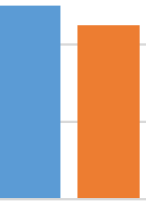

Minor

Moderate

Severe

- Cervical Thoracic

Severity of NBD and Cause of SCI

$100.00 \%$

$80.00 \%$

$60.00 \%$

$40.00 \%$

$20.00 \%$

$0.00 \%$

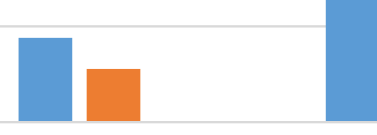

Very minor

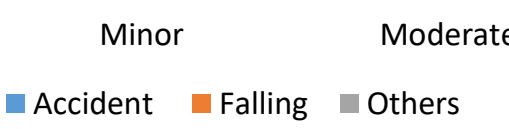

Figure 1. Severity of Neurogenic Bowel Dysfunction in Patients With Different LOI and Cause of Injury.

Table 3. Cleveland Clinic Constipation System (CCCS) Results

\begin{tabular}{|c|c|c|c|c|c|c|}
\hline & \multicolumn{2}{|c|}{ Descriptive Results } & \multicolumn{2}{|c|}{ Univariate Linear Regression to TSI } & \multicolumn{2}{|c|}{ Univariate Linear Regression to LOI } \\
\hline & Mean & SD & $\begin{array}{l}\text { Standardized } \\
\text { Coefficients } \\
\text { (Beta) }\end{array}$ & $\boldsymbol{P}$ & $\begin{array}{l}\text { Standardized } \\
\text { Coefficients } \\
\text { (Beta) }\end{array}$ & $\boldsymbol{P}$ \\
\hline Frequency of bowel movements & 1.06 & 0.78 & 0.253 & 0.070 & -0.086 & 0.556 \\
\hline Difficulty: painful evacuation effort & 1.90 & 1.50 & 0.338 & $0.014^{*}$ & 0.061 & 0.680 \\
\hline Completeness: feeling incomplete & 1.92 & 1.31 & 0.039 & 0.780 & 0.126 & 0.393 \\
\hline Pain: abdominal pain & 1.46 & 1.43 & 0.082 & 0.560 & -0.253 & 0.082 \\
\hline Time: minutes in lavatory per attempt & 1.90 & 1.54 & 0.295 & $0.037^{*}$ & 0.192 & 0.200 \\
\hline Assistance: type of assistance & 1.08 & 0.93 & 0.203 & 0.147 & -0.462 & $0.001^{* *}$ \\
\hline $\begin{array}{l}\text { Failure: unsuccessful attempts for } \\
\text { evacuation per } 24 \text { hours }\end{array}$ & 0.83 & 1.35 & -0.196 & 0.161 & 0.232 & 0.112 \\
\hline History: duration of constipation & 0.38 & 0.63 & 0.392 & $0.004^{* *}$ & 0.121 & 0.409 \\
\hline $\begin{array}{l}\text { Cleveland Clinic constipation system } \\
\text { score }\end{array}$ & 10.46 & 5.36 & 0.279 & $0.044^{*}$ & 0.005 & 0.971 \\
\hline
\end{tabular}

Abbreviations: SCl, spinal cord injury; TSI, time since injury; LOI, level of injury.

${ }^{*} P$ value $<0.05 ;{ }^{* *} P$ value $<0.01$ 
the subscales, difficulty: painful evacuation effort (B $=0.338, P=0.014)$, time: minutes spent in lavatory per attempt $(\mathrm{B}=0.295, P=0.037)$, and history: duration of constipation was positively correlated with the TSI (Table 3).

\section{Regression to the Level of Injury}

The analysis revealed no significant association between the CCCS score and the LOI. However, among the subscales, assistance: type of assistance was negatively associated with LOI $(B=-0.462, P=0.001$, Table 3$)$.

\section{Discussion}

In this study, we observed that some aspects of NBD were associated with certain characteristics of SCI. After regression analysis was done, certain subscales of the NBD score and the CCCS score were found to have a linear correlation with LOI and TSI. Moreover, the cause of injury and LOI were associated with different patterns of NBD severity among the patients' population. To summarize, this study denotes that possible associations exist between bowel issues of patients with SCI face and variables such as LOI, TSI, and the cause of injury. These associations need to be further investigated with greater sample sizes, more reliable study designs, and more meticulous statistical methods.

With respect to the associations with the interval since SCI, we see that among the subscales of the NBD score, there was a significant association between the frequency of defecation and the TSI: the more time had passed since the injury, the less frequent were the bowel movements. This observation is unparalleled in studies similar studies. A questionnaire-based study by Lynch et al that aimed to describe the bowel function of spinal cord injured (SCI) patients had previously shown that there were no changes in bowel motion frequency with TSI for either the patients or the controls. ${ }^{17}$ If we consider that the enteric nervous system of the large bowel undergoes pathological alterations after SCI, and that these alterations worsen as time passes since the injury, this observation can be explained. This is evidenced by a study by White and Holmes which reported the changes of the colonic neuromuscular junctions in rats with experimental high-thoracic (T3) SCI. The results showed a significant decrease in colonic enteric neuron density that became more pronounced as more time passed since the occurrence of injury. ${ }^{18}$ This reasoning implies that TSI should be associated with more invasive methods for stimulating defecation, which is proven true by the significant association observed between the time passed and digital stimulation or evacuation of the anorectum. Furthermore, frequent digital stimulation can be the reason why perianal skin problems are positively associated with TSI. On the other hand, as bowel movements occur more frequently when less time has passed since SCI, the possibility of fecal incontinence can be expected to increase, as it does in the results of the study. Additionally, when the CCCS score is regressed to interval since SCI, we can observe a clear association, implying that the effects of constipation tend to become grave as time passes since the occurrence of SCI. The subscales "difficulty: painful evacuation", "time: minutes spent in lavatory per attempt", "history: duration of constipation" were also positively associated with the TSI. These correlations back up the possibility that the alterations of the bowel become more prominent as time passes. Also, in the case of constipation, a report by Salvioli and colleagues revealed that some patients with chronic SCI suffering from constipation have an abnormally high sigmoid colon compliance, have a significantly lower tolerance of lower sigmoid distension, and presented an abnormal pattern of visceral sensation referral, ${ }^{19}$ all implying that a type of neural remodeling has taken place.

Considering the effects of LOI, when the subscales of NBD score are examined, we can see that a higher LOI is significantly correlated with uneasiness, headache, or perspiration, complaints commonly associated with painful evacuation and constipation. Moreover, regular use of drops against constipation was also associated with a higher LOI. A similar pattern can be observed within the CCCS scales: the type of assistance was negatively correlated with the type of assistance, indicating that patients with a higher LOI were more in need of intervention to cope with constipation. These results are not surprising since a higher LOI usually causes a higher extent of disruption in the autonomic nervous system. ${ }^{20}$ Lesions above T1 are associated with prolonged mouthto-cecum transit time, but transit times in paraplegics are comparable to normal. ${ }^{21}$ Moreover, in a questionnairebased study involving 90 patients. De Looze and colleagues observed that constipation seemed to be more frequent in those who were tetraplegic compared with paraplegics, but the difference was not significant. Yet, paraplegics with a lesion below T10 suffered significantly less from constipation. ${ }^{22}$

With respect to the patterns of NBD severity, regardless of the fact that the LOI is cervical or thoracic, a similar Gaussian distribution is observed in the population. The mean is located on the minor severity group in both cases, and the variances are not wildly different. Also, the fact that a some of the thoracic patients reported severe NBD is probably due to the larger size of the thoracic subgroup. On the other hand, when the severity is investigated according to the cause, patterns are not as similar. A majority of patients injured by falling experienced minor NBD, while patients affected by accidents tended to 
experience a wider range of NBD severity with a higher variance among the patients. This could be because accidents per se encompass a more diverse spectrum of traumas compared to falling, owing to the fact that when a road accident occurs, there are multiple factors that can influence the severity of injury: the type of the vehicle (car versus motorcycle), the position of the victim's seat inside the car, the mechanism of the injury (whether the injury was sustained during the collision or the rollover), the time interval between the sustenance of injury and receiving medical care, etc. ${ }^{23}$ The patients that have been injured because of other causes all experience moderate NBD, which suggests that the characteristics of this group should be investigated in more detail in further studies.

Limitations of the study should be acknowledged. The cross-sectional nature of the study means that we only investigated the prevalence of factors and their associations. In order to make more definite conclusions, study designs that investigate the incidence of the problems and their associations with SCI characteristics are recommended. Also, each of the SCI characteristics investigated could serve as a confounding variable in the analysis of other characteristics, and it is useful to stratify the patients in future studies, or to analyze the characteristics against each other to detect and be wary of possible confounders. Lastly, larger samples sized can be considered when designing further studies.

\section{Conclusion}

Certain characteristics of SCI, namely interval since injury, LOI, and the cause of injury, are correlated with different aspects of bowel function of patients with SCI. To better define and observe these associations, studies with larger samples and prospective designs are warranted.

\section{Conflict of Interest Disclosures}

The authors declare that they have no conflict of interests.

\section{Ethical Statement}

All ethical principles were considered in this study. The participants were informed about the purpose of the study and its implementation stages and signed informed consent form before the involvement in the study.

\section{References}

1. Onose G, Anghelescu A, Muresanu DF, Padure L, Haras MA, Chendreanu CO, et al. A review of published reports on neuroprotection in spinal cord injury. Spinal Cord. 2009;47(10):716-26. doi: 10.1038/sc.2009.52.

2. Kwon BK, Tetzlaff W, Grauer JN, Beiner J, Vaccaro AR. Pathophysiology and pharmacologic treatment of acute spinal cord injury. Spine J. 2004;4(4):451-64. doi: 10.1016/j. spinee.2003.07.007.
3. Sekhon LH, Fehlings MG. Epidemiology, demographics, and pathophysiology of acute spinal cord injury. Spine (Phila Pa 1976). 2001;26(24 Suppl):S2-12. doi: 10.1097/00007632-200112151-00002.

4. Yuan YM, He C. The glial scar in spinal cord injury and repair. Neurosci Bull. 2013;29(4):421-35. doi: 10.1007/ s12264-013-1358-3.

5. O'Shea TM, Burda JE, Sofroniew MV. Cell biology of spinal cord injury and repair. J Clin Invest. 2017;127(9):3259-70. doi: $10.1172 /$ jci90608.

6. Filipp ME, Travis BJ, Henry SS, Idzikowski EC, Magnuson SA, Loh MY, et al. Differences in neuroplasticity after spinal cord injury in varying animal models and humans. Neural Regen Res. 2019;14(1):7-19. doi: 10.4103/16735374.243694.

7. Ahuja CS, Wilson JR, Nori S, Kotter MRN, Druschel C, Curt A, et al. Traumatic spinal cord injury. Nat Rev Dis Primers. 2017;3:17018. doi: 10.1038/nrdp.2017.18.

8. Stiens SA, Bergman SB, Goetz LL. Neurogenic bowel dysfunction after spinal cord injury: clinical evaluation and rehabilitative management. Arch Phys Med Rehabil. 1997;78(3 Suppl):S86-102. doi: 10.1016/s00039993(97)90416-0.

9. Krogh K, Mosdal C, Gregersen H, Laurberg S. Rectal wall properties in patients with acute and chronic spinal cord lesions. Dis Colon Rectum. 2002;45(5):641-9. doi: 10.1007/ s10350-004-6261-6.

10. Keshavarzian A, Barnes WE, Bruninga K, Nemchausky B, Mermall H, Bushnell D. Delayed colonic transit in spinal cord-injured patients measured by indium-111 Amberlite scintigraphy. Am J Gastroenterol. 1995;90(8):1295-300.

11. MacDonagh R, Sun WM, Thomas DG, Smallwood R, Read NW. Anorectal function in patients with complete supraconal spinal cord lesions. Gut. 1992;33(11):1532-8. doi: 10.1136/gut.33.11.1532.

12. Krogh K, Christensen P, Sabroe S, Laurberg S. Neurogenic bowel dysfunction score. Spinal Cord. 2006;44(10):625-31. doi: 10.1038/sj.sc.3101887.

13. Levi R, Hultling C, Nash MS, Seiger A. The Stockholm spinal cord injury study: 1 . Medical problems in a regional SCI population. Paraplegia. 1995;33(6):308-15. doi: 10.1038/sc.1995.70.

14. Glickman S, Kamm MA. Bowel dysfunction in spinalcord-injury patients. Lancet. 1996;347(9016):1651-3. doi: 10.1016/s0140-6736(96)91487-7.

15. Cao Y, Krause JS. The association between secondary health conditions and indirect costs after spinal cord injury. Spinal Cord. 2021;59(3):306-10. doi: 10.1038/s41393-02000567-4.

16. Agachan F, Chen T, Pfeifer J, Reissman P, Wexner SD. A constipation scoring system to simplify evaluation and management of constipated patients. Dis Colon Rectum. 1996;39(6):681-5. doi: 10.1007/bf02056950.

17. Lynch AC, Wong C, Anthony A, Dobbs BR, Frizelle FA. Bowel dysfunction following spinal cord injury: a 
description of bowel function in a spinal cord-injured population and comparison with age and gender matched controls. Spinal Cord. 2000;38(12):717-23. doi: 10.1038/ sj.sc. 3101058 .

18. White AR, Holmes GM. Anatomical and functional changes to the colonic neuromuscular compartment after experimental spinal cord injury. J Neurotrauma. 2018;35(9):1079-90. doi: 10.1089/neu.2017.5369.

19. Salvioli B, Bazzocchi G, Barbara G, Stanghellini V, Cremon C, Menarini M, et al. Sigmoid compliance and visceral perception in spinal cord injury patients. Eur J Gastroenterol Hepatol. 2012;24(3):340-5. doi: 10.1097/ MEG.0b013e32834fbf73.

20. Lujan HL, DiCarlo SE. Direct comparison of cervical and high thoracic spinal cord injury reveals distinct autonomic and cardiovascular consequences. J Appl Physiol (1985). 2020;128(3):554-64. doi: 10.1152/japplphysiol.00721.2019.

21. Rajendran SK, Reiser JR, Bauman W, Zhang RL, Gordon SK, Korsten MA. Gastrointestinal transit after spinal cord injury: effect of cisapride. Am J Gastroenterol. 1992;87(11):1614-7.

22. De Looze D, Van Laere M, De Muynck M, Beke R, Elewaut A. Constipation and other chronic gastrointestinal problems in spinal cord injury patients. Spinal Cord. 1998;36(1):63-6. doi: 10.1038/sj.sc.3100531.

23. Fakharian E, Mohammadzadeh M, Saberi HR, Fazel MR, Rejali M, Akbari $\mathrm{H}$, et al. Spinal injury resulting from car accident: Focus to prevention. Asian J Neurosurg. 2017;12(2):180-4. doi: 10.4103/1793-5482.152110. 